Article

\title{
On Nonlinear Fractional Difference Equation with Delay and Impulses
}

\author{
Rujira Ouncharoen ${ }^{1}$, Saowaluck Chasreechai ${ }^{2, *}$ and Thanin Sitthiwirattham ${ }^{3, *}$ (])
}

1 Research Center in Mathematics and Applied Mathematics, Department of Mathematics, Faculty of Science, Chiang Mai University, Chiang Mai 50200, Thailand; rujira.o@cmu.ac.th

2 Department of Mathematics, Faculty of Applied Science, King Mongkut's University of Technology North Bangkok, Bangkok 10800, Thailand

3 Mathematics Department, Faculty of Science and Technology, Suan Dusit University, Bangkok 10300, Thailand

* Correspondence: saowaluck.c@sci.kmutnb.ac.th (S.C.); thanin_sit@dusit.ac.th (T.S.)

Received: 12 May 2020; Accepted: 5 June 2020; Published: 8 June 2020

check for updates

\begin{abstract}
In this paper, we establish the existence results for a nonlinear fractional difference equation with delay and impulses. The Banach and Schauder's fixed point theorems are employed as tools to study the existence of its solutions. We obtain the theorems showing the conditions for existence results. Finally, we provide an example to show the applicability of our results.
\end{abstract}

Keywords: fractional difference equations; delay; impulses; existence

JEL Classification: 39A05; 39A12

\section{Introduction}

Discrete fractional calculus studies have been an interesting field of present day, because some real-world phenomena are described by using fractional difference operators (see papers [1-3] and the references therein). Basic knowledge of fractional difference calculus can be found in [4]. The extension of this field can be found in [5-37] and references cited therein.

For the development of the fractional difference equations theory, which is the discrete case of fractional differential equations, there are still few publications. However, there are some recent papers studying fractional difference equations with delay. In 2017, Kaewwisetkul et al. [38] studied boundary value problems for Caputo fractional functional difference equations with delay. In 2018, Wu et al. [39] proposed the finite-time stability of discrete fractional delay systems, Alzabut et al. [40] studied nonlinear delay fractional difference equations with applications on the discrete fractional Lotka-Volterra competition model, Alzabut et al. [41] investigated the application on the uniqueness of solutions for nonlinear delay fractional difference system, and Luo et al. [42] considered the uniqueness and finite-time stability of solutions for a class of nonlinear fractional delay difference systems.

In particular, the fractional difference equations with delay and impulses have not been studied extensively. In 2018, Wu et al. [43] studied a linear fractional delay difference equations with impulse. These results are incentives for research. In this paper, we propose a nonlinear fractional difference equation with delay and impulses of the form:

$$
\begin{aligned}
& \Delta_{C}^{\alpha} u(t)=F\left[t+\alpha-1, u_{t+\alpha-1}, \Delta^{\beta} u(t+\alpha-\beta)\right], t \in \mathbb{N}_{0, T}, t+\alpha-1 \neq t_{k} \\
& u\left(t_{k}\right)=I_{k}\left(u_{t_{k}-1}\right), \quad k=1,2, \ldots, p, t_{k+1}-t_{k} \geq 2, \\
& u(t+\alpha-1)=\psi(t+\alpha-1), \quad t \in \mathbb{N}_{-r, 0}, r \in \mathbb{N}_{0, T+1},
\end{aligned}
$$


where $\mathbb{N}_{0, T}:=\{0,1, \ldots, T\}, \alpha, \beta \in(0,1), \Delta u\left(t_{k}\right)=u\left(t_{k}+1\right)-u\left(t_{k}\right), t_{0}=\alpha-1<t_{1}<t_{2}<\ldots<$ $t_{p}<T+\alpha, F \in C\left(\mathbb{N}_{\alpha-1, T+\alpha} \times C_{r} \times \mathbb{R}, \mathbb{R}\right), I_{k}: C_{r} \rightarrow \mathbb{R}$ and $\psi$ is an element of the space:

$$
C_{r}^{+}(\alpha-1):=\left\{\psi \in C_{r}: \psi(\alpha-1)=0, \Delta_{C}^{\beta} \psi(s-\beta+1)=0, s \in \mathbb{N}_{\alpha-r-1, \alpha-1}\right\} .
$$

For $r \in \mathbb{N}_{0, T+1}$, let $C_{r}$ be the Banach space of all continuous functions $\psi: \mathbb{N}_{\alpha-r-1, \alpha-1} \rightarrow \mathbb{R}$ with the norm:

$$
\|\psi\|_{C_{r}}=\max _{s \in \mathbb{N}_{\alpha-r-1, \alpha-1}}|\psi(s)| .
$$

If $u: \mathbb{N}_{\alpha-r-1, \alpha-1} \rightarrow \mathbb{R}$, then for any $t \in \mathbb{N}_{\alpha-1, T+\alpha}$, we define the element $u_{t}$ of $C_{r}$ as,

$$
u_{t}(\theta)=u(t+\theta) \text { for } \theta \in \mathbb{N}_{-r, 0} .
$$

We aim to prove the existence results to the problem of Equation (1) by using the Banach and Schauder's fixed point theorems. Finally, we present an example in the last section.

\section{Preliminaries}

In this section, we recall some notations, definitions, and lemmas used in the main results.

Definition 1. The generalized falling function is defined by:

$$
t^{\underline{\alpha}}:=\frac{\Gamma(t+1)}{\Gamma(t+1-\alpha)}
$$

If $t+1-\alpha$ is a pole of the Gamma function and $t+1$ is not a pole, then $t^{\underline{\alpha}}=0$.

Definition 2. For $\alpha>0$ and $f$ defined on $\mathbb{N}_{a}:=\{a, a+1, \ldots\}$, the $\alpha$-order fractional sum of $f$ is defined by:

$$
\Delta^{-\alpha} f(t):=\frac{1}{\Gamma(\alpha)} \sum_{s=a}^{t-\alpha}(t-\sigma(s)) \frac{\alpha-1}{2} f(s),
$$

where $t \in \mathbb{N}_{a+\alpha}$ and $\sigma(s)=s+1$.

Definition 3. For $\alpha>0, N \in \mathbb{N}$ is satisfied with $0 \leq N-1<\alpha<N$ and $f$ defined on $\mathbb{N}_{a}$, the $\alpha$-order Riemann-Liouville fractional difference of $f$ is defined by:

$$
\Delta^{\alpha} f(t):=\Delta^{N} \Delta^{-(N-\alpha)} f(t)=\frac{1}{\Gamma(-\alpha)} \sum_{s=a}^{t+\alpha}(t-\sigma(s)) \frac{-\alpha-1}{-1} f(s),
$$

where $t \in \mathbb{N}_{a+N-\alpha}$. The $\alpha$-order Caputo fractional difference of $f$ is defined by:

$$
\Delta_{C}^{\alpha} f(t):=\Delta^{-(N-\alpha)} \Delta^{N} f(t)=\frac{1}{\Gamma(N-\alpha)} \sum_{s=a}^{t-(N-\alpha)}(t-\sigma(s))^{N-\alpha-1} \Delta^{N} f(s),
$$

where $t \in \mathbb{N}_{a+N-\alpha}$. If $\alpha=N$, then $\Delta^{\alpha} f(t)=\Delta_{C}^{\alpha} f(t)=\Delta^{N} f(t)$.

Lemma 1. [5] Assume that $\alpha>0$ and $f$ defined on $\mathbb{N}_{a}$. Then,

$$
\Delta^{-\alpha} \Delta_{C}^{\alpha} y(t)=y(t)+C_{0}+C_{1}(t-a)^{1}+C_{2}(t-a)^{2}+\ldots+C_{N-1}(t-a)^{\frac{N-1}{}},
$$

for some $C_{i} \in \mathbb{R}, 0 \leq i \leq N-1$ and $0 \leq N-1<\alpha \leq N$. 
Next, we aim to find a solution of the linear variant of the mixed problem in Equation (1) as follows.

Lemma 2. Let $\alpha \in(0,1), h \in C\left(\mathbb{N}_{\alpha-1, T+\alpha}, \mathbb{R}\right), I_{k}: C_{r} \rightarrow \mathbb{R}$ and $\psi \in C_{r}^{+}(\alpha-1)$ be given. Then the problem

$$
\begin{aligned}
& \Delta_{C}^{\alpha} u(t)=h(t+\alpha-1), \quad t \in \mathbb{N}_{0, T}:=\{0,1, \ldots, T\}, t+\alpha-1 \neq t_{k} \\
& u\left(t_{k}\right)=I_{k}\left(u_{t_{k}-1}\right), \quad k=1,2, \ldots, p, t_{k+1}-t_{k} \geq 2, \\
& u(t+\alpha-1)=\psi(t+\alpha-1), \quad t \in \mathbb{N}_{-r, 0}, r \in \mathbb{N}_{0, T+1},
\end{aligned}
$$

has the unique solution which is in a form:

$$
u(t)= \begin{cases}\frac{1}{\Gamma(\alpha)} \sum_{s=t_{0}}^{t-1}(t-s+\alpha-2)^{\frac{\alpha-1}{h} h(s),} & t \in \mathbb{N}_{t_{0}, t_{1}} \\ \sum_{i=1}^{k} I_{i}\left(u_{t_{i}-1}\right)+\frac{1}{\Gamma(\alpha)} \sum_{i=1}^{k} \sum_{s=t_{i-1}}^{t_{i}-1}\left(t_{i}-s+\alpha-2\right)^{\frac{\alpha-1}{h}} h(s) & \\ \quad+\frac{1}{\Gamma(\alpha)} \sum_{s=t_{k}}^{t-1}(t-s+\alpha-2)^{\frac{\alpha-1}{h}} h(s), & t \in \mathbb{N}_{t_{k}+1, t_{k+1}} \\ \varphi(t), & t \in \mathbb{N}_{\alpha-r-1, \alpha-1}\end{cases}
$$

where $\Delta u\left(t_{k}\right)=u\left(t_{k}+1\right)-u\left(t_{k}\right), t_{0}=\alpha-1<t_{1}<t_{2}<\ldots<t_{p}<T+\alpha$.

Proof. For $t \in \mathbb{N}_{t_{0}, t_{1}}$, taking the fractional sum of order $\alpha$ for Equation (2) and from Lemma 1, we have:

$$
u(t)=\varphi(\alpha-1)+\frac{1}{\Gamma(\alpha)} \sum_{s=0}^{t-\alpha}(t-\sigma(s))^{\frac{\alpha-1}{h}} h(s+\alpha-1) .
$$

From $\varphi(\alpha-1)=0$, we can write Equation (4) as:

$$
u(t)=\frac{1}{\Gamma(\alpha)} \sum_{s=t_{0}}^{t-1}(t-s+\alpha-2) \frac{\alpha-1}{h} h(s) .
$$

By substituting $t=t_{1}$ into Equation (5), we have:

$$
u\left(t_{1}\right)=\frac{1}{\Gamma(\alpha)} \sum_{s=t_{0}}^{t_{1}-1}\left(t_{1}-s+\alpha-2\right)^{\frac{\alpha-1}{2}} h(s) .
$$

If $t \in \mathbb{N}_{t_{1}+1, t_{2}}$, then we get"

$$
\begin{aligned}
u(t) & =u\left(t_{1}+1\right)+\frac{1}{\Gamma(\alpha)} \sum_{s=t_{1}}^{t-1}(t-s+\alpha-2)^{\frac{\alpha-1}{2}} h(s) \\
& =\Delta u\left(t_{1}\right)+u\left(t_{1}\right)+\frac{1}{\Gamma(\alpha)} \sum_{s=t_{1}}^{t-1}(t-s+\alpha-2)^{\frac{\alpha-1}{}} h(s) .
\end{aligned}
$$


Substiting $u\left(t_{1}\right)$ from Equation (6) to above equation, we obtain:

$$
\begin{aligned}
u(t)= & I_{1}\left(u_{t_{1}-1}\right)+\frac{1}{\Gamma(\alpha)} \sum_{s=t_{0}}^{t_{1}-1}\left(t_{1}-s+\alpha-2\right) \frac{\alpha-1}{h} h(s) \\
& +\frac{1}{\Gamma(\alpha)} \sum_{s=t_{1}}^{t-1}(t-s+\alpha-2) \frac{\alpha-1}{h} h(s) .
\end{aligned}
$$

If $t \in \mathbb{N}_{t_{2}+1, t_{3}}$, then we have:

$$
\begin{aligned}
u(t) & =u\left(t_{2}+1\right)+\frac{1}{\Gamma(\alpha)} \sum_{s=t_{2}}^{t-1}(t-s+\alpha-2)^{\frac{\alpha-1}{}} h(s) \\
& =\Delta u\left(t_{2}\right)+u\left(t_{2}\right)+\frac{1}{\Gamma(\alpha)} \sum_{s=t_{2}}^{t-1}(t-s+\alpha-2)^{\frac{\alpha-1}{h}} h(s) .
\end{aligned}
$$

Substiting $u\left(t_{2}\right)$ from Equation (7) to above equation, we obtain:

$$
\begin{aligned}
u(t)= & I_{2}\left(u_{t_{2}-1}\right)+\left[I_{1}\left(u_{t_{1}-1}\right)+\frac{1}{\Gamma(\alpha)} \sum_{s=t_{0}}^{t_{1}-1}\left(t_{1}-s+\alpha-2\right)^{\frac{\alpha-1}{h}} h(s)\right. \\
& \left.+\frac{1}{\Gamma(\alpha)} \sum_{s=t_{1}}^{t_{2}-1}\left(t_{2}-s+\alpha-2\right) \frac{\alpha-1}{h} h(s)\right]+\frac{1}{\Gamma(\alpha)} \sum_{s=t_{2}}^{t-1}(t-s+\alpha-2) \frac{\alpha-1}{h} h(s) .
\end{aligned}
$$

By using the recursive process, we obtain the solution $u(t)$ for $t \in \mathbb{N}_{t_{k}+1, t_{k+1}}(k=1,2, \ldots, p)$ as given by:

$$
\begin{aligned}
u(t)= & \sum_{i=1}^{k} I_{i}\left(u_{t_{i}-1}\right)+\frac{1}{\Gamma(\alpha)} \sum_{i=1}^{k} \sum_{s=t_{i-1}}^{t_{i}-1}\left(t_{i}-s+\alpha-2\right)^{\frac{\alpha-1}{}} h(s) \\
& +\frac{1}{\Gamma(\alpha)} \sum_{s=t_{k}}^{t-1}(t-s+\alpha-2)^{\frac{\alpha-1}{}} h(s) .
\end{aligned}
$$

Obviously, for each $t \in \mathbb{N}_{\alpha-r-1, \alpha-1}$, we have $u(t)=\varphi(t)$.

\section{Existence and Uniqueness Result}

In this section, we employ the Banach fixed point theorem to consider the existence and uniqueness result for the problem in Equation (1). Define the Banach space:

$$
\mathcal{X}:=\left\{u: u \in C\left(\mathbb{N}_{\alpha-r-1, T+\alpha}, \mathbb{R}\right), \Delta^{\beta} u \in C\left(\mathbb{N}_{\alpha-\beta-r, T+\alpha-\beta+1}, \mathbb{R}\right), 0<\beta<1\right\}
$$

with the norm defined by:

$$
\|u\|_{\mathcal{X}}=\|u\|+\left\|\Delta^{\beta} u\right\|,
$$

where $\|u\|=\max _{t \in \mathbb{N}_{\alpha-r-1, T+\alpha}}|u(t)|$ and $\left\|\Delta^{\beta} u\right\|=\max _{t \in \mathbb{N}_{\alpha-r-1, T+\alpha}}\left|\Delta^{v} u(t-\beta+1)\right|$.

In view of the definitions of $u_{t}$ and $\psi$, we have:

$$
u_{\alpha-1}=u_{\alpha-1}(\theta)=u(\theta+\alpha-1)=\psi(\theta+\alpha-1) \text { for } \theta \in \mathbb{N}_{-r, 0} .
$$


Thus, we obtainL

$$
u(t)=\psi(t) \text { for } t \in \mathbb{N}_{\alpha-r-1, \alpha-1} .
$$

Next, define an operator $\mathcal{T}: \mathcal{X} \rightarrow \mathcal{X}$ as:

$$
(\mathcal{T} u)(t):= \begin{cases}\frac{1}{\Gamma(\alpha)} \sum_{s=t_{0}}^{t-1}(t-s+\alpha-2)^{\frac{\alpha-1}{}} F\left[s, u_{s}, \Delta^{\beta} u(s-\beta+1)\right], & t \in \mathbb{N}_{t_{0}, t_{1}} \\ \sum_{i=1}^{k} I_{i}\left(u_{t_{i}-1}\right)+\frac{1}{\Gamma(\alpha)} \sum_{i=1}^{k} \sum_{s=t_{i-1}}^{t_{i}-1}\left(t_{i}-s+\alpha-2\right)^{\frac{\alpha-1}{}} F\left[s, u_{s}, \Delta^{\beta} u(s-\beta+1)\right] & \\ \quad+\frac{1}{\Gamma(\alpha)} \sum_{s=t_{k}}^{t-1}(t-s+\alpha-2)^{\frac{\alpha-1}{2}} F\left[s, u_{s}, \Delta^{\beta} u(s-\beta+1)\right], & t \in \mathbb{N}_{t_{k}+1, t_{k+1}} \\ \varphi(t), & t \in \mathbb{N}_{\alpha-r-1, \alpha-1}\end{cases}
$$

where $k=1,2, \ldots, p, t_{k+1}-t_{k} \geq 2, t_{0}=\alpha-1<t_{1}<t_{2}<\ldots<t_{p}<T+\alpha$.

Firstly, we provide some basic knowledge that is used in this section as follows.

Definition 4. A mapping $S$ from a subset $M$ of a Banach space $X$ into $X$ is called a contraction mapping (or simply a contraction) if there exists a positive number $\alpha<1$ such that:

$$
\|S(x)-S(y)\|_{X} \leq \alpha\|x-y\|_{X} \text { for all } x, y \in M .
$$

Lemma 3. [44] (Banach fixed point theorem) Let $M$ be a closed subset of a Banach space X and let $S$ be a contraction mapping from $M$ into $M$. Then there exists a uniquez $\in M$ such that $S(z)=z$.

If one can prove that $\mathcal{T}$ has fixed point, we can conclude that the problem of Equation (1) has a solution.

Theorem 1. Assume the following properties:

(H1) There exists a constant $\ell>0$ such that:

$$
\left|F\left[t, u_{1}, u_{2}\right]-F\left[t, v_{1}, v_{2}\right]\right| \leq \ell\left(\left|u_{1}-v_{1}\right|+\left|u_{2}-v_{2}\right|\right) ，
$$

for each $u_{1}, v_{1} \in C_{r}$ and $u_{2}, v_{2} \in \mathbb{R}$.

(H2) There exists a constant $\lambda>0$ such that

$$
\left|I_{k}\left(u_{t_{k-1}}\right)-I_{k}\left(v_{t_{k-1}}\right)\right| \leq \lambda\left|u_{t}-v_{t}\right|,
$$

for each $u_{t}, v_{t} \in C_{r}$ and $k=1,2, \ldots, p$.

(H3) $\left[\frac{p(p+1)}{2} \lambda+\ell\left(\frac{p(p+1)}{2}+1\right) \frac{(T+\alpha) \underline{\alpha}}{\Gamma(\alpha+1)}\right]\left[1+\frac{(T+\alpha-\beta-1) \underline{-\beta}}{\Gamma(1-\beta)}\right]<1$.

Then, the problem of Equation (1) has an unique solution.

Proof. We will show that $\mathcal{T}$ is a contraction. Letting,

$$
|\mathcal{H}(u-v)|(t)=\left|F\left[t, u_{t}, \Delta^{\beta} u(t-\beta+1)\right]-F\left[t, v_{t}, \Delta^{\beta} v(t-\beta+1)\right]\right|,
$$


for each $t \in \mathbb{N}_{\alpha-1, T+\alpha}$, we obtain:

$$
\begin{aligned}
|(\mathcal{T} u)(t)-(\mathcal{T} v)(t)| \leq & \sum_{i=1}^{p}\left|I_{i}\left(u_{t_{i}-1}\right)-I_{i}\left(v_{t_{i}-1}\right)\right|+\frac{1}{\Gamma(\alpha)} \sum_{i=1}^{k} \sum_{s=t_{i-1}}^{t_{i}-1}\left(t_{i}-s+\alpha-2\right)^{\frac{\alpha-1}{}}|\mathcal{H}(u-v)|(s) \\
& +\frac{1}{\Gamma(\alpha)} \sum_{s=t_{k}}^{t-1}(t-s+\alpha-2)^{\frac{\alpha-1}{}}|\mathcal{H}(u-v)|(s) \\
\leq & \frac{p(p+1)}{2} \lambda\left|u_{t}-v_{t}\right|+\frac{\ell\left(\left|u_{t}-v_{t}\right|+\left|\Delta^{\beta} u(t-\beta+1)-\Delta^{\beta} v(t-\beta+1)\right|\right)}{\Gamma(\alpha+1)} \times \\
& \left\{\left(\frac{p(p+1)}{2}+1\right)(T+\alpha)^{\underline{\alpha}}\right\} \\
\leq & {\left[\frac{p(p+1)}{2} \lambda+\ell\left(\frac{p(p+1)}{2}+1\right) \frac{(T+\alpha)^{\underline{\alpha}}}{\Gamma(\alpha+1)}\right]\|u-v\|_{\mathcal{X}} . }
\end{aligned}
$$

Taking the fractional difference of order $\beta$ for Equation (13) and substituting $t=t-\beta+1$, we get:

$$
\begin{aligned}
& \left(\Delta^{\beta} \mathcal{T} u\right)(t-\beta+1)
\end{aligned}
$$

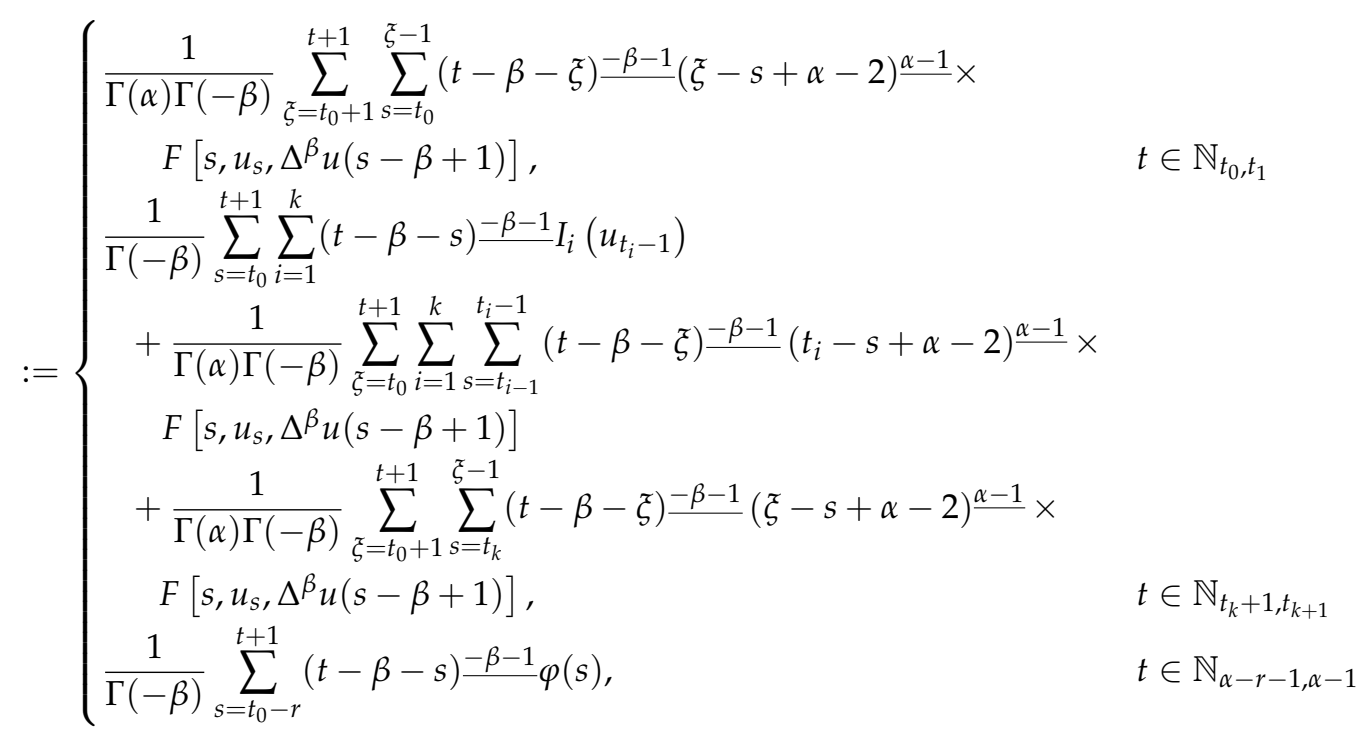

For each $t \in \mathbb{N}_{\alpha-1, T+\alpha}$, we obtain:

$$
\begin{aligned}
& \left|\left(\Delta^{\beta} \mathcal{T} u\right)(t-\beta+1)-\left(\Delta^{\beta} \mathcal{T} v\right)(t-\beta+1)\right| \\
& \leq \frac{1}{\Gamma(-\beta)} \sum_{s=t_{0}}^{T+\alpha+1}(T+\alpha-\beta-s) \frac{-\beta-1}{2} \sum_{i=1}^{p}\left|I_{i}\left(u_{t_{i}-1}\right)-I_{i}\left(v_{t_{i}-1}\right)\right|+\frac{1}{\Gamma(\alpha) \Gamma(-\beta)} \times
\end{aligned}
$$

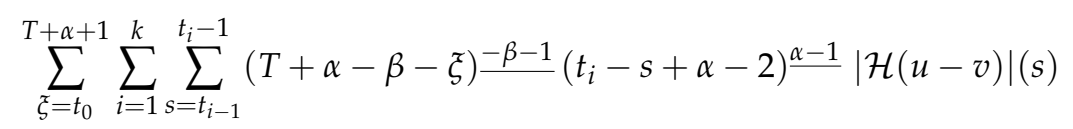

$$
\begin{aligned}
& +\frac{1}{\Gamma(\alpha) \Gamma(-\beta)} \sum_{\xi=t_{0}+1}^{T+\alpha+1} \sum_{s=t_{k}}^{\xi-1}(T+\alpha-\beta-\xi) \frac{-\beta-1}{}(\xi-s+\alpha-2)^{\frac{\alpha-1}{2}}|\mathcal{H}(u-v)|(s) \\
& \leq \frac{p(p+1)}{2} \lambda\left|u_{t}-v_{t}\right|+\frac{\ell\left(\left|u_{t}-v_{t}\right|+\left|\Delta^{\beta} u(t-\beta+1)-\Delta^{\beta} v(t-\beta+1)\right|\right)}{\Gamma(\alpha+1)} \times \\
& \left\{\left(\frac{p(p+1)}{2}+1\right)(T+\alpha)^{\underline{\alpha}}\right\} \\
& \leq \frac{(T+\alpha-\beta-1) \underline{-\beta}}{\Gamma(1-\beta)}\left[\frac{p(p+1)}{2} \lambda+\ell\left(\frac{p(p+1)}{2}+1\right) \frac{(T+\alpha)^{\underline{\alpha}}}{\Gamma(\alpha+1)}\right]\|u-v\|_{\mathcal{X}} .
\end{aligned}
$$


Obviously, for each $t \in \mathbb{N}_{\alpha-r-1, \alpha-1}$, we get $|(\mathcal{T} u)(t)-(\mathcal{T} v)(t)|=0$.

Therefore, we have:

$$
\begin{aligned}
& \|(\mathcal{T} u)-(\mathcal{T} v)\|_{\mathcal{X}} \\
\leq & {\left[\frac{p(p+1)}{2} \lambda+\ell\left(\frac{p(p+1)}{2}+1\right) \frac{(T+\alpha)^{\underline{\alpha}}}{\Gamma(\alpha+1)}\right]\left[1+\frac{(T+\alpha-\beta-1)^{-\beta}}{\Gamma(1-\beta)}\right]\|x-y\|_{\mathcal{X}} . }
\end{aligned}
$$

By (H3), it implies that $\mathcal{T}$ is a contraction. Therefore, by Banach fixed point theorem, $\mathcal{T}$ has a fixed point which is a unique solution of the problem in Equation (1).

\section{Existence of at Least One Solution}

In this section, we also present the existence of at least one solution of Equation (1) by using the Schauder's fixed point theorem. Firstly, we provide some basic knowledge that is used in this section as follows.

Lemma 4. [44] (Arzelá-Ascoli theorem) A set of function in $C[a, b]$ with the sup norm, is relatively compact if and only it is uniformly bounded and equicontinuous on $[a, b]$.

Lemma 5. [44] A bounded set in $\mathbb{R}^{n}$ is relatively compact, a closed bounded set in $\mathbb{R}^{n}$ is compact.

Lemma 6. [45] (Schauder's fixed point theorem) Let $(D, d)$ be a complete metric space, $U$ be a closed convex subset of $D$, and $T: D \rightarrow D$ be the map such that the set $T u: u \in U$ is relatively compact in $D$. Then the operator $T$ has at least one fixed point $u^{*} \in U: T u^{*}=u^{*}$.

The following notations are defined for using in the sequel.

$$
\begin{aligned}
& \Theta=\max _{t \in \mathbb{N}_{\alpha-r-1, \alpha-1}}\left\{\left(\frac{1}{2} p(p+1)+1\right) \frac{(T+\alpha)^{\underline{\alpha}}}{\Gamma(\alpha+1)} \phi(t)\right\} \\
& \widetilde{\Theta}=\max _{t \in \mathbb{N}_{\alpha-r-1, \alpha-1}}\left\{\left(\frac{1}{2} p(p+1)+1\right) \frac{(T+\alpha)^{\underline{\alpha}}(T+\alpha-\beta+1) \underline{-\beta}}{\Gamma(\alpha+1) \Gamma(1-\beta)} \phi(t)\right\} \\
& \mathrm{Y}=\left(\frac{1}{2} p(p+1)+1\right) \frac{(T+\alpha)^{\underline{\alpha}}}{\Gamma(\alpha+1)}\left[1+\frac{(T+\alpha-\beta+1) \underline{-\beta}}{\Gamma(1-\beta)}\right] .
\end{aligned}
$$

Theorem 2. Assume the following properties:

(H4) There exists a nonnegative function $\phi \in C\left(\mathbb{N}_{\alpha-1, T+\alpha}\right)$ such that:

$$
|F[t, x, y]| \leq \phi(t)+\lambda_{1}|x|^{\chi_{1}}+\lambda_{2}|y|^{\chi_{2}},
$$

for each $x \in C_{r}, y \in \mathbb{R}$ where $\lambda_{1}, \lambda_{2}$ are negative constants and $0<\chi_{1}, \chi_{2}<1$; or

(H5) there exists a nonnegative function $\phi \in C\left(\mathbb{N}_{\alpha-3, T+\alpha}\right)$ such that:

$$
|F[t, x, y]| \leq \phi(t)+\lambda_{1}|x|^{\chi_{1}}+\lambda_{2}|y|^{\chi_{2}},
$$

for each $x \in C_{r}, y \in \mathbb{R}$ where $\lambda_{1}, \lambda_{2}$ are negative constants and $\chi_{1}, \chi_{2}>1$.

Then boundary value problem of Equation (1) has at least one solution.

Proof. The proof is organized into three steps as follows. 
Step I. We verify that $\mathcal{T}$ map bounded sets into bounded sets. Let $\max \left|I_{k}\left(u_{t_{k}-1}\right)\right|=N$ for $k=1,2, \ldots, p$. Suppose that $(\mathrm{H} 4)$ holds, we choose a constant:

$$
R \geq \max \left\{3\left[\frac{1}{2} p(p+1) N \frac{(T+\alpha-\beta+1) \frac{1-\beta}{\Gamma(1-\beta)}}{\Gamma}+1\right]+\Theta+\widetilde{\Theta},\left(3 \lambda_{1} Y\right)^{\frac{1}{1-\chi_{1}}},\left(3 \lambda_{2} Y\right)^{\frac{1}{1-\chi_{2}}}\right\}
$$

and define the $\mathcal{P}=\{u \in \mathcal{X}:\|u\| \leq R, R>0\}$.

For any $u \in \mathcal{P}$, we have:

$$
\begin{aligned}
|(\mathcal{T} u)(t)| \leq & \sum_{i=1}^{p}\left|I_{i}\left(u_{t_{i}-1}\right)\right|+\frac{1}{\Gamma(\alpha)} \sum_{i=1}^{p} \sum_{s=t_{i-1}}^{t_{i}-1}\left(t_{i}-s+\alpha-2\right)^{\frac{\alpha-1}{\alpha}} \times \\
& {\left[\phi(s)+\lambda_{1}\left|u_{s}\right|^{\chi_{1}}+\lambda_{2}\left|\Delta^{\beta} u(s-\beta+1)\right|^{\chi_{2}}\right]+\frac{1}{\Gamma(\alpha)} \sum_{s=t_{k}}^{t-1}(t-s+\alpha-2)^{\frac{\alpha-1}{} \times} } \\
& {\left[\phi(s)+\lambda_{1}\left|u_{s}\right|^{\chi_{1}}+\lambda_{2}\left|\Delta^{\beta} u(s-\beta+1)\right|^{\chi_{2}}\right] } \\
\leq & \frac{1}{2} p(p+1) N+\Theta+\left(\frac{1}{2} p(p+1)+1\right) \frac{(T+\alpha)^{\underline{\alpha}}}{\Gamma(\alpha)}\left[\lambda_{1}\left|u_{s}\right| \chi_{1}+\lambda_{2}\left|\Delta^{\beta} u(s-\beta+1)\right|^{\chi_{2}}\right]
\end{aligned}
$$

and

$$
\begin{aligned}
& \left|\left(\Delta^{\beta} \mathcal{T} u\right)(t-\beta+1)\right| \leq \frac{1}{\Gamma(-\beta)} \sum_{s=\alpha-1}^{t+1}(t-\beta-s) \frac{-\beta-1}{L}\left[\sum_{i=1}^{p}\left|I_{i}\left(u_{t_{i}-1}\right)\right|\right] \\
& +\frac{1}{\Gamma(\alpha) \Gamma(-\beta)} \sum_{\xi=t_{0}}^{t+1} \sum_{i=1}^{p} \sum_{s=t_{i-1}}^{t_{i}-1}(t-\beta-\xi) \frac{-\beta-1}{}\left(t_{i}-s+\alpha-2\right)^{\frac{\alpha-1}{}} \times \\
& {\left[\phi(s)+\lambda_{1}\left|u_{s}\right| \chi_{1}+\lambda_{2}\left|\Delta^{\beta} u(s-\beta+1)\right|^{\chi_{2}}\right]} \\
& +\frac{1}{\Gamma(\alpha) \Gamma(-\beta)} \sum_{\xi=t_{0}+1}^{t+1} \sum_{s=t_{k}}^{\xi-1}(t-\beta-\xi) \frac{-\beta-1}{-}(\xi-s+\alpha-2)^{\frac{\alpha-1}{}} \times \\
& {\left[\phi(s)+\lambda_{1}\left|u_{s}\right| \chi_{1}+\lambda_{2}\left|\Delta^{\beta} u(s-\beta+1)\right|^{\chi_{2}}\right]} \\
& \leq \frac{1}{2} p(p+1) N \frac{(T+\alpha-\beta+1) \frac{-\beta}{-\beta}}{\Gamma(1-\beta)}+\widetilde{\Theta}+\left(\frac{1}{2} p(p+1)+1\right) \times \\
& \frac{(T+\alpha) \underline{\alpha}(T+\alpha-\beta+1) \underline{-\beta}}{\Gamma(\alpha) \Gamma(1-\beta)}\left[\lambda_{1}\left|u_{s}\right| \chi_{1}+\lambda_{2}\left|\Delta^{\beta} u(s-\beta+1)\right|^{\chi_{2}}\right] \text {. }
\end{aligned}
$$

Hence, we have:

$$
\begin{aligned}
\|\mathcal{T} u\|_{\mathcal{X}} \leq & \frac{1}{2} p(p+1) N\left[\frac{(T+\alpha-\beta+1) \underline{-\beta}}{\Gamma(1-\beta)}+1\right]+\Theta+\widetilde{\Theta} \\
& +\mathrm{Y}\left[\lambda_{1}\left|u_{s}\right|^{\chi_{1}}+\lambda_{2}\left|\Delta^{\beta} u(s-\beta+1)\right|^{\chi_{2}}\right] \\
\leq & \frac{R}{3}+\mathrm{Y}\left[\lambda_{1}\left|u_{s}\right|{ }^{\chi_{1}}+\lambda_{2}\left|\Delta^{\beta} u(s-\beta+1)\right|^{\chi_{2}}\right] \\
\leq & \frac{R}{3}+\frac{R}{3}+\frac{R}{3}=R .
\end{aligned}
$$

This implies that $\mathcal{T}: \mathcal{P} \rightarrow \mathcal{P}$. 
For the second case, if (H5) holds, we choose a constant:

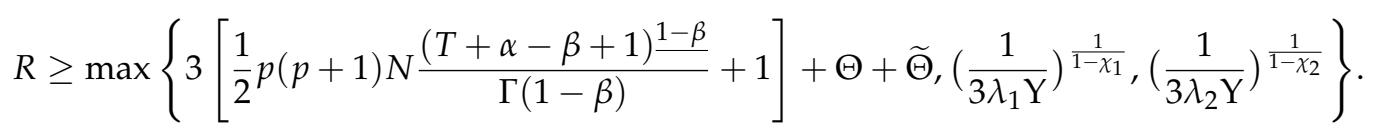

Similary, we find that:

$$
\|\mathcal{T} u(t)\|_{\mathcal{X}} \leq R,
$$

which implies that $\mathcal{T}: \mathcal{P} \rightarrow \mathcal{P}$.

Step II. It is obvious that the operator $\mathcal{T}$ is continuous on $\mathcal{P}$ since the continuity of $F$.

Step III. We prove that $\mathcal{T}$ is equicontinuous on $\mathcal{P}$. For any $\epsilon>0$, there exist positive constants $\delta_{1}=\max \left\{\delta_{11}, \delta_{12}\right\}, \delta_{2}$ and $\delta_{3}=\max \left\{\delta_{31}, \delta_{32}, \delta_{33}\right\}$, such that:

(i) for $\tau_{1}, \tau_{2} \in \mathbb{N}_{\alpha-1, T+\alpha}$ and $\tau_{1}<\tau_{2}$

$$
\begin{gathered}
\left|\tau_{2}^{\alpha}-\tau_{1}^{\alpha}\right|<\frac{\epsilon \Gamma(\alpha+1)}{2 M}, \text { whenever }\left|\tau_{2}-\tau_{1}\right|<\delta_{11} \\
\left|\left(\tau_{2}-\beta+1\right) \underline{-\beta}-\left(\tau_{1}-\beta+1\right) \underline{-\beta}\right|<\frac{\epsilon \Gamma(\alpha+1) \Gamma(1-\beta)}{2 M} \text {, whenever }\left|t_{2}-t_{1}\right|<\delta_{12}
\end{gathered}
$$

(ii) for $\tau_{1}, \tau_{2} \in \mathbb{N}_{\alpha-r-1, \alpha-1}$ and $\tau_{1}<\tau_{2}$

$$
\left|\psi\left(\tau_{2}\right)-\psi\left(\tau_{2}\right)\right|<\epsilon, \text { whenever }\left|\tau_{2}-\tau_{1}\right|<\delta_{2} ;
$$

(iii) for $\tau_{1} \in \mathbb{N}_{\alpha-r-1, \alpha-1}$ and $\tau_{2} \in \mathbb{N}_{\alpha, T+\alpha}$

$$
\begin{gathered}
\left|\tau_{2}^{\alpha}\right|<\frac{\epsilon \Gamma(\alpha+1)}{3 M}, \text { whenever }\left|\tau_{2}-\tau_{1}\right|<\delta_{31}, \\
\left|\psi\left(\tau_{1}\right)\right|<\frac{\epsilon}{3 M}, \text { whenever }\left|\tau_{2}-\tau_{1}\right|<\delta_{32}, \\
\left|\left(\tau_{2}-\beta+1\right) \frac{-\beta}{-}\right|<\frac{\epsilon \Gamma(\alpha+1) \Gamma(1-\beta)}{3 M}, \text { whenever }\left|\tau_{2}-\tau_{1}\right|<\delta_{33} .
\end{gathered}
$$

Let $M=\max _{t \in \mathbb{N}_{\alpha-1, T+\alpha}}\left|F\left[t, u_{t}, \Delta^{\beta} u(t-\beta+1)\right]\right|$. Then, for $u \in \mathcal{P}$ and $\tau_{1}, \tau_{2} \in \mathbb{N}_{\alpha-r-1, T+\alpha}$.

Case 1. If $\tau_{1}, \tau_{2} \in \mathbb{N}_{t_{0}, t_{1}} \cup \mathbb{N}_{t_{k}+1, t_{k+1}}, t_{k+1}-t_{k} \geq 2, k=1,2, \ldots, p$, and $\tau_{1}<\tau_{2}$, we obtain:

$$
\begin{aligned}
\left|(\mathcal{T} u)\left(\tau_{2}\right)-(\mathcal{T} u)\left(\tau_{1}\right)\right| & \leq \frac{M}{\Gamma(\alpha)}\left|\sum_{s=t_{k}}^{\tau_{2}-1}\left(\tau_{2}-s+\alpha-2\right) \frac{\alpha-1}{-\tau_{1}-1}-\sum_{s=t_{k}}\left(\tau_{1}-s+\alpha-1\right) \frac{\alpha-1}{}\right| \\
& \leq \frac{M}{\Gamma(\alpha+1)}\left|\tau_{2}^{\alpha}-\tau_{1}^{\alpha}\right| \\
& \leq \frac{\epsilon}{2}
\end{aligned}
$$


and

$$
\begin{aligned}
\mid\left(\Delta^{\beta} \mathcal{T} u\right)\left(\tau_{2}-\beta+1\right) & -\left(\Delta^{\beta} \mathcal{T} u\right)\left(\tau_{1}-\beta+1\right) \mid \\
\leq & \frac{M}{\Gamma(\alpha) \Gamma(-\beta)} \mid \sum_{\xi=t_{0}}^{\tau_{2}+1} \sum_{s=t_{k}}^{\xi-1}\left(\tau_{2}-\beta-\xi\right) \underline{-\beta-1}(\xi-s+\alpha-2)^{\underline{\alpha-1}} \\
& -\sum_{\xi=t_{0}}^{\tau_{1}+1=\sum_{s=t_{k}}^{\xi-1}}\left(\tau_{1}-\beta-\xi\right) \frac{-\beta-1}{\underline{\xi}}(\xi-s+\alpha-2)^{\underline{\alpha-1}} \mid \\
\leq & \frac{M}{\Gamma(\alpha+1) \Gamma(1-\beta)}\left|\left(\tau_{2}-\beta+1\right) \underline{-\beta}-\left(\tau_{1}-\beta+1\right) \underline{-\beta}\right| \\
\leq & \frac{\epsilon}{2} .
\end{aligned}
$$

By Equations (25) and (26), it implies that:

$$
\left\|(\mathcal{T} u)\left(\tau_{2}\right)-(\mathcal{T} u)\left(\tau_{1}\right)\right\|_{\mathcal{X}} \leq \frac{\epsilon}{2}+\frac{\epsilon}{2}=\epsilon
$$

Case 2. If $\tau_{1}, \tau_{2} \in \mathbb{N}_{\alpha-r-1, \alpha-1}$ and $\tau_{1}<\tau_{2}$, we obtain:

$$
\begin{aligned}
\left|(\mathcal{T} u)\left(\tau_{2}\right)-(\mathcal{T} u)\left(\tau_{1}\right)\right| & =\left|\psi\left(\tau_{2}\right)-\psi\left(\tau_{1}\right)\right|<\epsilon, \\
\text { and } \quad\left|\left(\Delta^{\beta} \mathcal{T} u\right)\left(\tau_{2}\right)-\left(\Delta^{\beta} \mathcal{T} u\right)\left(\tau_{1}\right)\right| & =\left|\left(\Delta^{\beta} \mathcal{T} \psi\right)\left(\tau_{2}\right)-\left(\Delta^{\beta} \mathcal{T} \psi\right)\left(\tau_{1}\right)\right|=0 .
\end{aligned}
$$

By Equations (28) and (29), it implies that:

$$
\left\|(\mathcal{T} u)\left(\tau_{2}\right)-(\mathcal{T} u)\left(\tau_{1}\right)\right\|_{\mathcal{X}}<\epsilon+0=\epsilon .
$$

Case 3. If $\tau_{1} \in \mathbb{N}_{\alpha-r-1, \alpha-1}$ and $\tau_{2} \in \mathbb{N}_{t_{0}+1, t_{1}} \cup \mathbb{N}_{t_{k}+1, t_{k+1}}, t_{k+1}-t_{k} \geq 2, k=1,2, \ldots, p$, we obtain:

$$
\begin{aligned}
\left|(\mathcal{T} u)\left(\tau_{2}\right)-(\mathcal{T} u)\left(\tau_{1}\right)\right| & \leq\left|(\mathcal{T} u)\left(\tau_{2}\right)-(\mathcal{T} u)(\alpha-1)\right|+\left|(\mathcal{T} u)(\alpha-1)-(\mathcal{T} u)\left(\tau_{1}\right)\right| \\
& \leq(\mathcal{T} u)\left(\tau_{2}\right)+\left|\psi\left(\tau_{1}\right)\right| \\
& \leq \frac{M}{\Gamma(\alpha+1)}\left|\tau_{2}^{\frac{\alpha}{2}}\right|+\left\|\varphi\left(\tau_{1}\right)\right\|_{C_{r}} \\
& <\frac{\epsilon}{3}+\frac{\epsilon}{3}=\frac{2 \epsilon}{3}
\end{aligned}
$$

and

$$
\begin{aligned}
\left|\left(\Delta^{\beta} \mathcal{T} u\right)\left(\tau_{2}\right)-\left(\Delta^{\beta} \mathcal{T} u\right)\left(\tau_{1}\right)\right| & \leq\left|\left(\Delta^{\beta} \mathcal{T} u\right)\left(\tau_{2}\right)-0\right| \\
& \leq \frac{M}{\Gamma(\alpha) \Gamma(-\beta)} \sum_{\xi=t_{0}}^{\tau_{2}+1} \sum_{s=t_{k}}^{\xi-1}\left(\tau_{2}-\beta-\xi\right) \frac{-\beta-1}{(\xi-s+\alpha-2) \frac{\alpha-1}{}} \\
& \leq \frac{M}{\Gamma(\alpha+1) \Gamma(1-\beta)}\left|\left(\tau_{2}-\beta+1\right) \underline{-\beta}\right| \\
& \leq \frac{\epsilon}{3} .
\end{aligned}
$$

By Equations (31) and (32), it implies that:

$$
\left\|(\mathcal{T} u)\left(\tau_{2}\right)-(\mathcal{T} u)\left(\tau_{1}\right)\right\|_{\mathcal{X}}<\frac{2 \epsilon}{3}+\frac{\epsilon}{3}=\epsilon
$$

From Steps I to III and the Arzelá-Ascoli theorem, we can conclude that $\mathcal{T}: \mathcal{X} \rightarrow \mathcal{X}$ is completely continuous. Therefore, the problem in Equation (1) has at least one solution by Schauder's fixed point theorem. 


\section{An Example}

Consider the following fractional difference boundary value problem:

$$
\begin{aligned}
\Delta_{C}^{\frac{1}{2}} u(t) & =F\left[t-\frac{1}{2}, u_{t-\frac{1}{2}}, \Delta^{\frac{3}{2}} u\left(t-\frac{1}{4}\right)\right], \quad t \in \mathbb{N}_{0,10}, t-\frac{1}{2} \neq t_{k}, k=1,2,3 \\
\Delta u\left(t_{k}\right) & =\frac{1}{k+100} \sin \left|u\left(t_{k}-1\right)\right|, t_{k}=\frac{1}{2}+2 k, \\
u\left(-\frac{1}{2}\right) & =0 .
\end{aligned}
$$

Here $\alpha=\frac{1}{2}, \beta=\frac{2}{3}, T=5, p=3$.

(i) Let $F\left[t, u_{t}, \Delta^{\beta} u_{t-\beta+1}\right]=\frac{\left|u_{t}\right|+\left|\Delta^{\frac{2}{3}} u\left(t+\frac{1}{3}\right)\right|}{(t+100)^{3}\left[1+\left|u_{t}\right|+\left|\Delta^{\frac{2}{3}} u\left(t+\frac{1}{3}\right)\right|\right]}$.

For $t \in \mathbb{N}_{-\frac{1}{2}, \frac{1}{2}}$, we have:

$$
\left|F\left[t, u_{t}, \Delta^{\beta} u\right]-F\left[t, v_{t}, \Delta^{\beta} v\right]\right| \leq \frac{8}{7880599}\left[\left|u_{t}-v_{t}\right|+\left|\Delta^{\frac{2}{3}} u\left(t+\frac{1}{3}\right)-\Delta^{\frac{2}{3}} v\left(t+\frac{1}{3}\right)\right|\right] .
$$

So, (H1) holds with $\ell=\frac{8}{7880599}$.

For all $u, v \in \mathcal{X}$ and $k=1,2,3$, we have:

$$
\left|I_{k}(u)-I_{k}(v)\right| \leq \frac{1}{101}|u-v| .
$$

So, (H2) holds with $\lambda=\frac{1}{101}$.

We can show that (H3) holds with:

$$
\left[\frac{p(p+1)}{2} \lambda+\ell\left(\frac{p(p+1)}{2}+1\right) \frac{(T+\alpha)^{\underline{\alpha}}}{\Gamma(\alpha+1)}\right]\left[1+\frac{(T+\alpha-\beta-1) \underline{-\beta}}{\Gamma(1-\beta)}\right] \approx 0.06401<1 .
$$

Therefore, by Theorem 1, the boundary value problem of Equation (34) has an unique solution.

(ii) Let $F\left[t, u_{t}, \Delta^{\beta} u_{t-\beta+1}\right]=t^{2}+\frac{e^{-t}}{2(t+1)^{3}}\left|u_{t}\right|^{\chi_{1}}+\frac{e^{-2(t+1)}}{3}\left|\Delta^{\frac{2}{3}} u_{t+\frac{1}{3}}\right|^{\chi_{2}}$.

For $t \in \mathbb{N}_{-\frac{1}{2}, \frac{1}{2}}$, we have:

$$
\left|F\left[t, u_{t}, \Delta^{\beta} u_{t-\beta+1}\right]\right| \leq\left(\frac{21}{2}\right)^{\underline{2}}+\frac{4}{\sqrt{e}}\left|u_{t}\right|^{\chi_{1}}+\frac{1}{3 e}\left|\Delta^{\frac{2}{3}} u\left(t+\frac{1}{3}\right)\right|^{\chi_{2}} .
$$

Thus $|\phi(t)| \leq \frac{441}{4}, \lambda_{1}=\frac{4}{\sqrt{e}}, \lambda_{2}=\frac{1}{3 e}$. We can show that (H4) is satisfied for $0<\chi_{1}, \chi_{2}<1$, and (H5) is satisfied for $\chi_{1}, \chi_{2}>1$. Therefore, by Theorem 2, the boundary value problem in Equation (34) has at least one solution.

\section{Conclusions}

We established the conditions for the existence and uniqueness of a solution for a nonlinear fractional difference equation with delay and impulses in Equation (1) by using the Banach fixed point theorem, and the conditions of at least one solution by using the Schauder's fixed point theorem. Our problem contained both delay and impulses, which is a new idea. 
Author Contributions: Conceptualization, R.O. and S.C.; Formal analysis, R.O. and S.C.; Funding acquisition, S.C.; Investigation, R.O.; Methodology, R.O., S.C., and T.S.; Writing-original draft, R.O., S.C., and T.S.; Writing-review and editing, R.O., S.C., and T.S. All authors have read and agreed to the published version of the manuscript.

Funding: This research was funded by King Mongkut's University of Technology North Bangkok. Contract No. KMUTNB-61-GOV-D-65.

Acknowledgments: This research was supported by Chiang Mai University.

Conflicts of Interest: The authors declare no conflict of interest regarding the publication of this paper.

\section{References}

1. Wu, G.C.; Baleanu, D. Discrete fractional logistic map and its chaos. Nonlinear Dyn. 2014, 75, $283-287$. [CrossRef]

2. Wu, G.C.; Baleanu, D. Chaos synchronization of the discrete fractional logistic map. Signal Process. 2014, 102, 96-99. [CrossRef]

3. Wu, G.C.; Baleanu, D.; Xie, H.P.; Chen F.L. Chaos synchronization of fractional chaotic maps based on stability results. Physica A 2016, 460, 374-383. [CrossRef]

4. Goodrich, C.S.; Peterson, A.C. Discrete Fractional Calculus; Springer: New York, NY, USA, 2015.

5. Abdeljawad, T. On Riemann and Caputo fractional differences, Comput. Math. Appl. 2011, 62, 1602-1611.

6. Abdeljawad, T. Dual identities in fractional difference calculus within Riemann. Adv. Differ. Equ. 2013, 2013, 36. [CrossRef]

7. Abdeljawad, T. On delta and nabla Caputo fractional differences and dual identities. Discrete. Dyn. Nat. Soc. 2013, 2013, 406910. [CrossRef]

8. Atici, F.M.; Eloe, P.W. Two-point boundary value problems for finite fractional difference equations. J. Differ. Equ. Appl. 2011, 17, 445-456. [CrossRef]

9. Atici, F.M.; Eloe, P.W. A transform method in discrete fractional calculus. Int. J. Differ. Equ. 2007, 2, $165-176$.

10. Atici, F.M.; Eloe, P.W. Initial value problems in discrete fractional calculus. Proc. Amer. Math. Soc. 2009, 137, 981-989. [CrossRef]

11. Agarwal, R.P.; Leanu, D.; Rezapour, S.; Salehi, S. The existence of solutions for some fractional finite difference equations via sum boundary conditions, Adv. Differ. Equ. 2014, 2014, 282. [CrossRef]

12. Goodrich, C.S. On discrete sequential fractional boundary value problems. J. Math. Anal. Appl. 2012, 385, 111-124. [CrossRef]

13. Goodrich, C.S. On a discrete fractional three-point boundary value problem. J. Differ. Equ. Appl. 2012, 18, 397-415. [CrossRef]

14. Erbe, L.; Goodrich, C.S.; Jia, B.; Peterson, A. Survey of the qualitative properties of fractional difference operators: monotonicity, convexity, and asymptotic behavior of solutions. Adv. Differ. Equ. 2016, 2016, 43. [CrossRef]

15. Chen, Y.; Tang, X. Three difference between a class of discrete fractional and integer order boundary value problems. Commun. Nonlinear Sci. 2014, 19, 4057-4067. [CrossRef]

16. Lv, W.; Feng, J. Nonlinear discrete fractional mixed type sum-difference equation boundary value problems in Banach spaces. Adv. Differ. Equ. 2014, 2014, 184. [CrossRef]

17. Lv, W. Existence of solutions for discrete fractional boundary value problems witha $p$-Laplacian operator. Adv. Differ. Equ. 2012, 2012, 163. [CrossRef]

18. Jia, B.; Erbe, L.; Peterson, A. Two monotonicity results for nabla and delta fractional differences. Arch. Math. 2015, 104, 589-597. [CrossRef]

19. Jia, B.; Erbe, L.; Peterson, A. Convexity for nabla and delta fractional differences. J. Differ. Equ. Appl. 2015, 21, 360-373.

20. Ferreira, R.A.C. Existence and uniqueness of solution to some discrete fractional boundary value problems of order less than one. J. Differ. Equ. Appl. 2013, 19, 712-718. [CrossRef]

21. Ferreira, R.A.C.; Goodrich, C.S. Positive solution for a discrete fractional periodic boundary value problem. Dyn. Contin. Discrete Impuls. Syst. Ser. A Math. Anal. 2012, 19, 545-557.

22. Kang, S.G.; Li, Y.; Chen, H.Q. Positive solutions to boundary value problems of fractional difference equations with nonlocal conditions. Adv. Differ. Equ. 2014, 2014, 7. [CrossRef] 
23. Dong, W.; Xu, J.; Regan, D.O. Solutions for a fractional difference boundary value problem. Adv. Differ. Equ. 2013, 2013, 319. [CrossRef]

24. Holm, M. Sum and difference compositions in discrete fractional calculus. Cubo 2011, 13, 153-184. [CrossRef]

25. Sitthiwirattham, T.; Tariboon J.; Ntouyas S.K. Existence results for fractional difference equations with three-point fractional sum boundary conditions. Discrete. Dyn. Nat. Soc. 2013, 2013, 104276. [CrossRef]

26. Sitthiwirattham, T.; Tariboon, J.; Ntouyas, S.K. Boundary value problems for fractional difference equations with three-point fractional sum boundary conditions. Adv. Differ. Equ. 2013, 2013, 296. [CrossRef]

27. Sitthiwirattham, T. Existence and uniqueness of solutions of sequential nonlinear fractional difference equations with three-point fractional sum boundary conditions. Math. Method. Appl. Sci. 2015, 38, 2809-2815. [CrossRef]

28. Sitthiwirattham, T. Boundary value problem for $p$-Laplacian Caputo fractional difference equations with fractional sum boundary conditions. Math. Method. Appl. Sci. 2016, 39, 1522-1534. [CrossRef]

29. Chasreechai, S.; Kiataramkul, C.; Sitthiwirattham, T. On nonlinear fractional sum-difference equations via fractional sum boundary conditions involving different orders. Math. Probl. Eng. 2015, 2015, 519072 [CrossRef]

30. Reunsumrit, J.; Sitthiwirattham, T. Positive solutions of three-point fractional sum boundary value problem for Caputo fractional difference equations via an argument with a shift. Positivity 2016, 20, 861-876. [CrossRef]

31. Reunsumrit, J.; Sitthiwirattham, T. On positive solutions to fractional sum boundary value problems for nonlinear fractional difference equations. Math. Method. Appl. Sci. 2016, 39, 2737-2751. [CrossRef]

32. Reunsumrit, R.; Sitthiwirattham, T. a New class of four-point fractional sum boundary value problems for nonlinear sequential fractional difference equations involving shift operators. Kragujevac J. Math. 2018, 42, 371-387. [CrossRef]

33. Chasreechai, S.; Sitthiwirattham, T. Existence results of initial value problems for hybrid fractional sum-difference equations. Discrete Dyn. Nat. Soc. 2018, 2018, 5268528. [CrossRef]

34. Chasreechai, S.; Sitthiwirattham, T. On separate fractional sum-difference boundary value problems with $n$-point fractional sum-difference boundary conditions via arbitrary different fractional orders. Mathematics 2019, 2019, 471. [CrossRef]

35. Kunnawuttipreechachan, E.; Promsakon, C.; Sitthiwirattham, T. Nonlocal fractional sum boundary value problem for a coupled system of fractional sum-difference equations. Dyn. Syst. Appl. 2019, 28, 73-92. [CrossRef]

36. Promsakon, C.; Chasreechai, S.; Sitthiwirattham, T. Positive solution to a coupled system of singular fractional difference equations with fractional sum boundary value conditions. Adv. Differ. Equ. 2019, 2019, 218. [CrossRef]

37. Soontharanon, J.; Chasreechai, S.; Sitthiwirattham, T. On a coupled system of fractional difference equations with nonlocal fractional sum boundary value conditions on the discrete half-line. Mathematics 2019, 2019, 256. [CrossRef]

38. Kaewwisetkul, B.; Sitthiwirattham, T. On nonlocal fractional sum-difference boundary value problems for Caputo fractional functional difference equations with delay. Adv. Differ. Equ. 2017, 2017, 219. [CrossRef]

39. Wu, G.C.; Baleanu, D.; Zeng, S.D. Finite-time stability of discrete fractional delay systems: Gronwall inequality and stability criterion. Commun. Nonlinear Sci. Numer. Simulat. 2018, 57, 299-308. [CrossRef]

40. Alzabut, J.; Abdeljawad, T.; Baleanu, D. Nonlinear delay fractional difference equations with applications on discrete fractional Lotka-Volterra competition model. J. Comput. Anal. Appl. 2018, 25, 889-898.

41. Alzabut, J.; Abdeljawad, T. A generalized discrete fractional Gronwall inequality and its application on the uniqueness of solutions for nonlinear delay fractional difference system. Appl. Anal. Discr. Math. 2018, 12, 36-48. [CrossRef]

42. Luo, D.; Luo, Z. Uniqueness and Novel Finite-Time Stability of Solutions for a Class of Nonlinear Fractional Delay Difference Systems. Discrete Dyn. Nat. Soc. 2018, 2018, 8476285. [CrossRef]

43. Wu, G.C.; Baleanu, D.; Huang, L.L. Novel Mittag-Leffler stability of linear fractional delay difference equations with impulse. Appl. Math. Lett. 2018, 82, 71-78. [CrossRef] 
44. Griffel, D.H. Applied Functional Analysis; Ellis Horwood Publishers: Chichester, UK, 1981.

45. Guo, D.; Lakshmikantham, V. Nonlinear Problems in Abstract Cone; Academic Press: Orlando, FL, USA, 1988. 